\title{
Ulceración esofágica por ingestión de doxiciclina
}

\section{Doxycycline induced esophageal ulceration}

\author{
Santiago Pereda ${ }^{1}$, Fernando M. Stengel ${ }^{2}$ y Juliana Förster Fernández ${ }^{3}$
}

\section{RESUMEN}

La ulceración esofágica por ingestión de doxiciclina es una de las causas más frecuentes de lesión esofágica. Ha sido subdiagnosticada y escasamente reconocida en dermatología. El dolor retroesternal, la odinofagia de aparición brusca y el antecedente de ingesta de doxiciclina u otros fármacos son características que facilitan su diagnóstico.
Puede presentar complicaciones serias, como hemorragias, estenosis y mediastinitis.

Palabras clave: ulceración esofágica, doxiciclina.

Dermatol. Argent. 2021, 27 (2): 75-77

\section{ABSTRACT}

Esophageal ulceration due to ingestion of doxycycline is one of the most frequent causes of esophageal injury. It has been underdiagnosed and scarcely recognized in dermatology. Retrosternal pain, sudden odynophagia and a history of doxycycline or other drugs intake are some of the characteristics that lead to diagnosis.
It may cause severe complications such as bleeding, stenosis and mediastinitis.

Key words: esophageal ulceration, doxycycline.

Dermatol. Argent. 2021, 27 (2): 75-77
${ }^{1}$ Estudiante, Facultad de Medicina, Universidad de Buenos Aires

${ }^{2}$ Médico Dermatólogo, Director Médico Buenos Aires Skin

${ }^{3}$ Médica Dermatóloga, Socia Fundadora de Buenos Aires Skin Ciudad Autónoma de Buenos Aires, Argentina
Contacto del autor: Fernando Stengel E-mail: fernando@stengeldermato.com.ar Fecha de trabajo recibido: 13/1/2021 Fecha de trabajo aceptado: 26/6/2021 Conflicto de interés: los autores declaran que no existe conflicto de interés.

\section{CASO CLÍNICO}

Una mujer de 23 ańos, sin antecedentes personales ni familiares significativos, consultó al dermatólogo por presentar un acné vulgar de grado moderado, con comedones y lesiones inflamatorias constituidas por pápulas y pústulas, con predominio facial. Recibió tratamientos tópicos, con respuesta parcial y temporaria, por lo que se le indicó doxiciclina $100 \mathrm{mg}$ por día, una hora antes o 2 horas después de las comidas, y medicación tópica con peróxido de benzoílo al 2,5\% y adapaleno al $0,1 \%$ en gel.
Dos a 3 horas luego de ingerir el segundo comprimido de doxiciclina, con pequeños sorbos de agua y momentos antes de acostarse a dormir, experimentó un dolor agudo e intenso en la región retroesternal media y odinofagia. Dadas la persistencia y la intensidad del cuadro, 8 horas después del inicio de los síntomas la paciente consultó en la guardia de emergencia. En el interrogatorio, surgió el antecedente de la ingesta previa de doxiciclina y se le realizó una endoscopia. 
En la videoendoscopia se observó una exulceración circunferencial del esófago con fibrina y tejido de granulación (Fotos 1 y 2). Los hallazgos en el estómago y el duodeno fueron normales. Se tomó una muestra para biopsia, en la cual se encontró una ulceración superficial de la mucosa con tejido de granulación inflamatorio e infiltrado fibrinoleucocitario, linfocitos, neutrófilos y escasos plasmocitos.

La paciente recibió omeprazol $40 \mathrm{mg} /$ día por vía oral durante 14 días y dieta líquida durante 3 días. $\mathrm{Al}$ cabo de 9 días se recuperó sin secuelas.

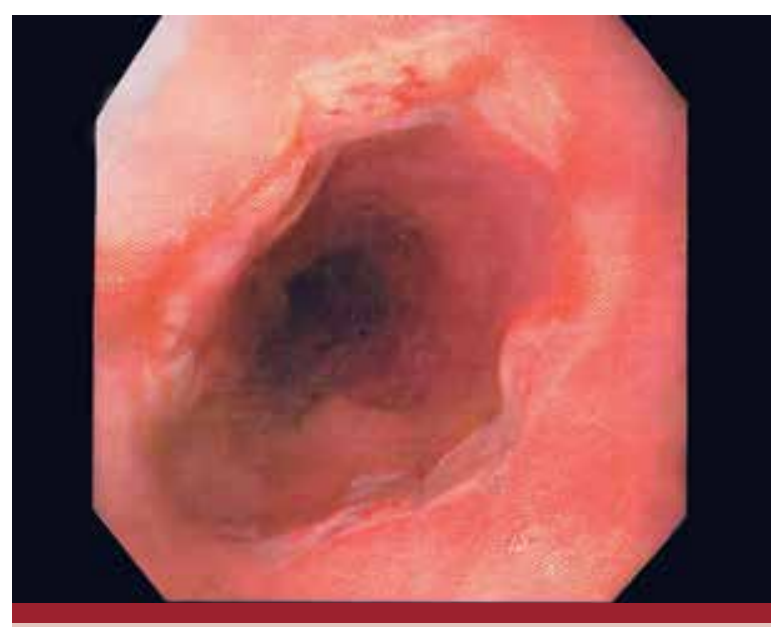

F0T0 1: En sitio de impronta esofágica del cayado aórtico, exulceración circunferencial con fibrina y presencia de tejido de granulación.

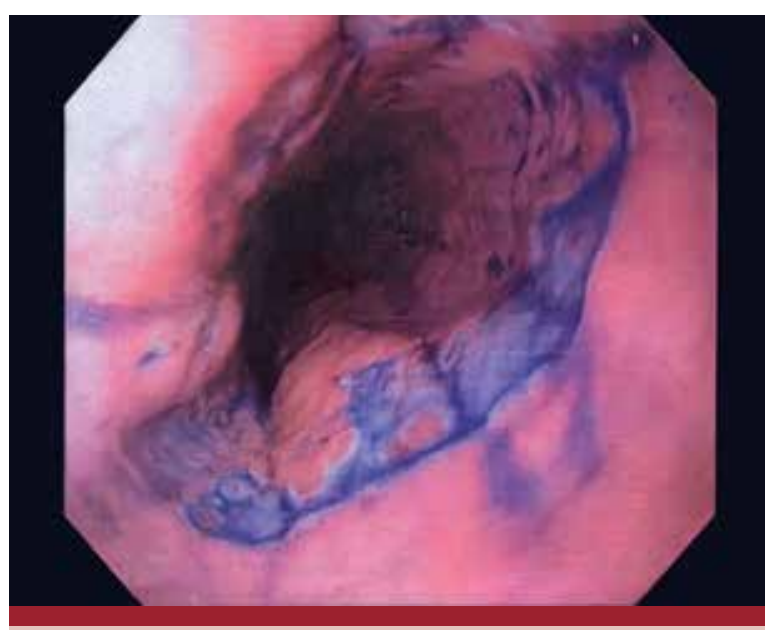

F0T0 2: Exulceración circunferencial: coloración con índigo carmín para realce del contraste.

\section{COMENTARIOS}

La úlcera gástrica por consumo de doxiciclina ha merecido escasa atención en nuestra especialidad, incluso en los tratados sobre el acné. En nuestra opinión, la esofagitis o ulceración esofágica de diferentes grados inducida por medicamentos es una entidad subdiagnosticada. Se han informado más de 1000 casos de úlceras esofágicas producidas por fármacos, con una incidencia aproximada de 4/100.000 pacientes y se vinculó este cuadro a más de 100 compuestos diferentes. Los antibióticos son los causantes del 50\% de los casos registrados. La doxiciclina fue el agente causal en el $27 \%$ del total ${ }^{1}$. Este fármaco tiene elevada biodisponibilidad, vida media y liposolubilidad, lo que determina una excelente penetración tisular. Además, su disolución es muy rápida, lo que puede derivar en un contacto más prolongado con la mucosa esofágica. Estos serían los factores protagonistas de la acción sobre la mucosa esofágica ${ }^{2,3}$.

La presentación del fármaco también es importante a la hora de interpretar el cuadro. Los comprimidos más grandes se retienen más a menudo en el esófago, así como aquellos que se presentan en forma de cápsulas ${ }^{4}$.

El cuadro es frecuente en los jóvenes sin patología esofágica, como en el caso analizado, pero los ancianos y las personas postradas corren mayor riesgo de sufrirlo.

Las lesiones esofágicas inducidas por fármacos se clasifican en dos grupos: lesiones autolimitadas, como las de la paciente, y esofagitis persistentes.

Los síntomas cardinales típicos -como en el caso comunicado- son el dolor retroesternal de aparición brusca, con odinofagia o no, que obliga a descartar problemas cardíacos agudos. Enfatizamos que las complicaciones severas agudas incluyen hemorragias, perforaciones y mediastinitis. A largo plazo, la estenosis y el carcinoma de esófago 5 .

En las personas sanas con sintomatología típica, ante un interrogatorio que revela la ingesta de una medicación sospechosa, no se realiza la esofagoscopia. Se aconseja el seguimiento clínico. La severidad inicial del cuadro, su persistencia o agravamiento, así como su ocurrencia en un huésped inmunodeprimido son indicaciones para la realización de una esofagoscopia instrumental. Es un estudio más sensible que la esofagoscopia con contraste de bario y permite realizar el cepillado o biopsia de las lesiones presentes ${ }^{1}$.

Como prevención, se aconseja ingerir la doxiciclina con no menos de $120 \mathrm{ml}$ de agua y en posición sentada o parada, no recostarse hasta 30 minutos después de la ingestión e interrogar a los pacientes sobre dismotilidad esofágica, estenosis previas o compresiones extrínsecas. En los pacientes postrados, conviene considerar otras alternativas terapéuticas ${ }^{6}$.

El tratamiento se basa en suspender la medicación sospechada e indicar una dieta líquida, con eventual hidratación parenteral en los casos severos. Puede administrarse una analgesia superficial con jaleas de xilocaína contra el dolor. En casos severos y durante el pe- 
ríodo agudo, está indicada la suspensión de sucralfato, con dosis inicial de $1 \mathrm{~g}$ cuatro veces por día, una hora antes de cada comida y al acostarse. Los inhibidores de la bomba de protones, como el omeprazol, deben ingerirse 30 minutos antes de la cena.

\section{BIBLIOGRAFÍA}

1. Kikendall JW. Pill esophagitis. J Clin Gastroenterol. 1999;28:298-305.

2. Shetty AK. Tetracyclines in pediatrics revisited. Clin Pediatr. 2002;41:203-209.

3. Gencosmanoglu R, Kurtkaya-Yapicier O, Tiftikci A, Avsar E, et ál. Midesophageal ulceration and candidiasis-associated distal esophagitis as two distinct clinical patterns of tetracycline or doxycyclineinduced esophageal injury. J Clin Gastroenterol 2004;38:484-489.

4. Hey H, Jørgensen F, Sørensen $\mathrm{K}$, Hasselbalch $\mathrm{H}$, et ál. Esophageal transit of
Presentamos el primer caso de la literatura dermatológica argentina de una úlcera esofágica aguda causada por la ingesta de doxiciclina. Señalamos sus características clínicas y enfatizamos los consejos para prevenir, reconocer y tratar el cuadro.

six commonly used tablets and capsules. BrMedJ. 1982;285:1717-1719.

5. Pace $F$, Antinori $S$, Repici $A$. What is new in esophageal injury (infection, drug-induced, caustic, stricture, perforation)? Curr Op Gastroenterol. 2009;25:372-379.

6. Katzka DA. Esophageal disorders caused by medications, trauma, and infection. En: Feldman M, Friedman LS, Brandt LJ. Sleisenger and Fortrand's Gastrointestinal and Liver Disease. Elsevier, Philadelphia, 2021:662.

\section{DERMATÓLOGOS JÓVENES}

\section{$\star$ Piense en... CARCINOMA BASOCELULAR SUPERFICIAL / Respuestas}

॥1 1

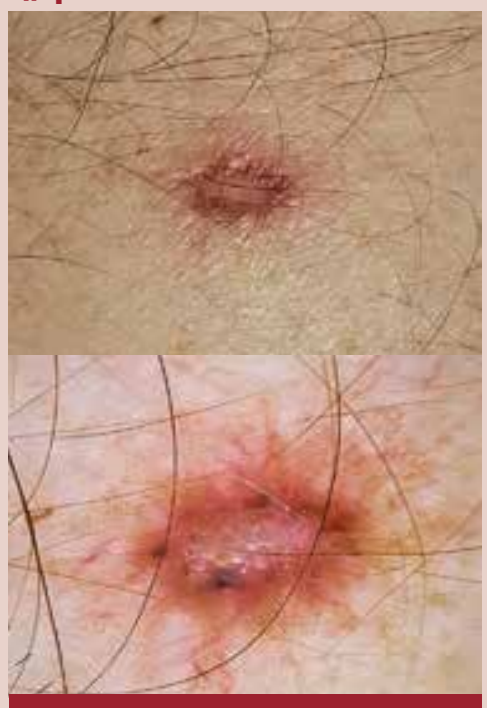

FOTO 1: Varón de 54 años. Antecedente de carcinoma basocelular en la mejilla izquierda.

DERMATOFIBROMA

- Tumor fibrohistiocítico benigno frecuente.

- Múltiples variantes clinicopatológicas.

- Clínica: nódulo duroelástico de color piel, marrón rojizo o marrón oscuro. Signo del "hoyuelo" o de Fitzpatrick.

- Se localiza con mayor frecuencia en los miembros inferiores.

- Dermatoscopia: el patrón más observado es un parche blanco central con retículo pigmentado en la periferia.

- Tratamiento: se puede realizar cirugía si presenta síntomas o molestias estéticas.

\section{1) 2}

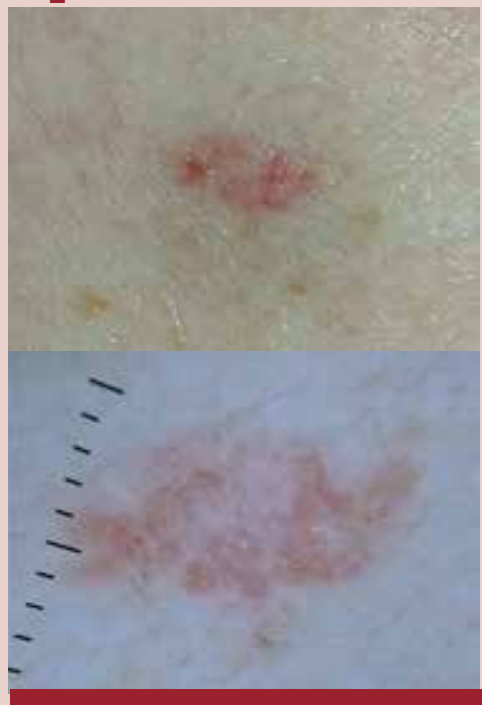

FOTO 2: Varón de 77 años. Antecedente de carcinomas basocelulares múltiples.

CARCINOMA BASOCELULARSUPERFICIAL

- El carcinoma basocelular (CBC) es el tumor maligno más frecuente. Deriva de queratinocitos basales. La variedad superficial es el segundo subtipo clínico más prevalente (30\%).

- Clínica: mácula o placa eritematosa, bien circunscripta.

- Se localiza principalmente en el tronco y los miembros.

- Dermatoscopia: estructuras blanco-rojizas brillantes, telangiectasias cortas y finas y ulceraciones superficiales mútiples.

- Tratamiento: cirugía.
॥ 3

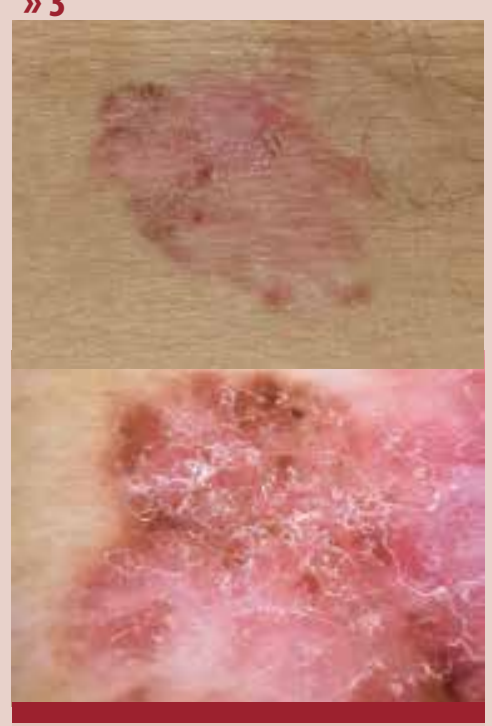

F0T0 3: Varón de 78 años. Antecedente de carcinomas basocelulares múltiples.

CARCINOMA ESPINOCELULAR INVASOR INDIFERENCIADO

- Neoplasia maligna derivada de queratinocitos del estrato espinoso. Es el segundo en frecuencia luego del CBC.

- Aumenta su incidencia a partir de los 40 años, principalmente en los varones.

- Clínica: pápula o placa hiperqueratósica que varía según el subtipo y la localización.

- Dermatoscopia: áreas sin estructura, hipopigmentadas, rosadas, color piel o blanquecinas. Vasos puntiformes 0 glomerulares, en horquilla y lineales, irregulares. Aperturas foliculares en "blanco de tiro", masa central de queratina y ulceración. De color marrón cuando es pigmentado.

- Tratamiento: cirugía.

\section{BIBLIOGRAFÍA}

1. Cives M, Mannavola F, Lospalluti L, Sergi MC, etál. Non-melanoma skin cancers: biological and clinical features. Int JMo/ Sci 2020;21:5394-5419.

2. Bandyopadhyay MR, Besra M, Dutta S, Sarkar S. Dermatofibroma: atypical presentations. Indian J Dermatol 2016;61:121.

3. Zaballos DP. Criterios dermatoscópicos de los tumores no melanocíticos. Med Cutan Iber Lat Am 2015;43:93-103.

4. Peralta R, Cohen Sabban EN, Cabo HA. Dermatoscopia de lesiones no melanocíticas: carcinoma de células escamosas in situ-enfermedad de Bowen. Forma pigmentada. Dermatol Argent 2013;19:369-370. 